\title{
Cellular response to glutamine and/or glucose deprivation in in vitro transformed human fibroblasts
}

\author{
ILARIA CHIODI, GIULIA PICCO, CAROLINA MARTINO and CHIARA MONDELLO \\ Institute of Molecular Genetics, Italian National Research Council (CNR), I-27100 Pavia, Italy
}

Received October 18, 2018; Accepted April 12, 2019

DOI: $10.3892 /$ or.2019.7125

\begin{abstract}
Neoplastic transformation is characterized by metabolic rewiring to sustain the elevated biosynthetic demands of highly proliferative cancer cells. To obtain the precursors for macromolecule biosynthesis, cancer cells avidly uptake and metabolize glucose and glutamine. Thus, targeting the availability or metabolism of these nutrients is an attractive anticancer therapeutic strategy. To improve our knowledge concerning how cancer cells respond to nutrient withdrawal, the response to glutamine and/or glucose starvation was studied in human in vitro transformed fibroblasts, deeply characterized at the cellular and molecular level. Concomitant starvation of both nutrients led to rapid loss of cellular adhesion ( $16 \mathrm{~h}$ after starvation), followed by cell death. Deprivation of glucose alone had the same effect, although at a later time ( $48 \mathrm{~h}$ after starvation), suggesting that glucose plays a key role in enabling cell attachment to the extracellular matrix. Glutamine deprivation did not induce rapid cell death, but caused a prolonged arrest of cellular proliferation; the cells started dying only $96 \mathrm{~h}$ after starvation. Before massive cell death occurred, the effects of all the starvation conditions were reversible. Autophagy activation was observed in cells incubated in the absence of glucose for more than $48 \mathrm{~h}$, while autophagy was not detected under the other starvation conditions. Markers of apoptotic cell death, such as caspase 3, caspase 9 and poly(ADP-ribose) polymerase 1 (PARP-1) proteolytic fragments, were not observed under any growth condition. Glucose and/or glutamine deprivation caused very rapid PARP-1 activation, with marked PARP-1 (poly-ADP) ribosylation and protein (poly-ADP) ribosylation. This activation was not due to starvation-induced DNA double-strand breaks, which appeared at the late stages of deprivation, when most cells died. Collectively, these results highlight a broad
\end{abstract}

Correspondence to: Dr Chiara Mondello, Institute of Molecular Genetics, Italian National Research Council (CNR), Via Abbiategrasso 207, I-27100 Pavia, Italy

E-mail: mondello@igm.cnr.it

Key words: glucose, glutamine, metabolism, apoptosis, autophagy, $\mathrm{H} 2 \mathrm{~A}$ histone family member $\mathrm{X}$, poly(ADP-ribose) polymerase, olaparib, cancer, transformed fibroblasts range of consequences of glucose and glutamine starvation, which may be taken into account when nutrient availability is used as a target for anticancer therapies.

\section{Introduction}

Metabolic reprogramming is a hallmark of cancer cells, supporting the acquisition and maintenance of malignant properties $(1,2)$. The high rate of proliferation characteristic of cancer cells requires an increase in nutrient uptake and anabolism in order to produce energy and precursors necessary for macromolecular biosynthesis. In this regard, glucose and glutamine metabolism has attracted particular interest, as these molecules are the main nutrients consumed by cancer cells (3). Despite the presence of oxygen, cancer cells mainly convert glucose into lactate through glycolysis, as first described by Otto Warburg (4), while glutamine is the major source of anaplerotic intermediates for the tricarboxylic acid (TCA) cycle, thus maintaining its functionality for the production of ATP (5). Through glucose and glutamine catabolism, cancer cells maintain a high pool of diverse intermediates that serve as building blocks for the biosynthesis of macromolecules. Moreover, oxidation of the carbon skeletons of glucose and glutamine allows cells to produce NADPH, which plays a crucial role in reductive biosynthetic reactions and helps to maintain cellular redox capacity (6). Analogously, glutamine is important for the production of glutathione, another antioxidant molecule (7).

In line with the multiple roles of glucose and glutamine in cancer cell physiology, deprivation of these nutrients impairs cellular viability, indicating that glucose and glutamine metabolism may be targeted for cancer therapy (8). Given that different cancer cell types can activate different responses to nutrient deprivation, such as apoptosis, autophagy or cell growth arrest, depending on genetic, epigenetic and environmental clues (9-12), it is important to improve our current understanding of cell behavior under conditions of starvation.

In the present study, well-characterized in vitro transformed cells were used to investigate the cellular and molecular response to glucose and/or glutamine starvation. Transformed cen3tel cells were derived from human fibroblasts immortalized by telomerase expression, which spontaneously became tumorigenic during propagation in culture (13-16). Following the transition from normal to transformed cells, genome-wide gene and miRNA expression profiles of cells representing 
different phases of transformation were examined by microarray analysis. Transformed cells exhibited changes in gene expression suggestive of a high usage of glutamine, such as overexpression of the $\mathrm{c}-M Y C$ oncogene, which is a regulator of several genes involved in glutamine metabolism (14). High levels of the c-Myc protein were paralleled by a reduced expression of miR-23a and miR-23b $(14,16)$, which negatively regulate glutaminase, the enzyme catalyzing the first step of glutamine metabolism with the conversion of glutamine to glutamate (17). Moreover, microarray analysis revealed that tumorigenic cells overexpressed the genes encoding the enzymes asparagine synthetase and aspartate aminotransferase, which are involved in non-essential amino acid biosynthesis from glutamine, as well as the enzymes pyruvate dehydrogenase kinase 1 and 3 , which inactivate pyruvate dehydrogenase, thereby preventing pyruvate from entering into the TCA cycle and downregulating aerobic respiration (National Center for Biotechnology Information database Gene Expression Omnibus; accession no. GSE15742).

The aim of the present study was to analyze the effect of glutamine and/or glucose deprivation on cellular viability in cen3tel cells at the latest stage of transformation, as well as the expression of markers associated with different cell death pathways and DNA double-strand break (DSB) induction.

\section{Materials and methods}

Cells and cell culture. The cen3tel cells used in this study belong to the cen3tel cellular system, which was developed from telomerase-immortalized fibroblasts that gradually underwent transformation during propagation in culture (13-16,18-20). Cen3tel cells underwent approximately 1,000 population doublings after telomerase immortalization. These cells are tumorigenic and metastatic in nude mice, overexpress c- $M Y C$ and carry a mutation in the TP53 codon 161, which has been used to confirm the identity of the cen3tel cells used in the present study $(14,15)$.

Cells were propagated in high-glucose Dulbecco's modified Eagle's medium (DMEM; Euroclone S.p.A, Pero, MI, Italy) supplemented with $10 \%$ newborn calf serum (EuroClone), $2 \mathrm{mM}$ glutamine (EuroClone) and non-essential amino acids $(0.1 \mathrm{mM}$ each L-alanine, $\mathrm{L}$-asparagine, $\mathrm{L}$-aspartic acid, L-glycine, L-serine, L-proline and L-glutamic acid, EuroClone). To determine the cellular response to nutrient deprivation, cen3tel cells were seeded in $3-\mathrm{cm}$ Petri dishes in complete medium at a concentration of $2-2.5 \times 10^{5}$ cells per dish and incubated at $37^{\circ} \mathrm{C}$. When the desired confluence was reached $\left(\sim 2-3 \times 10^{5}\right.$ cells $/ \mathrm{cm}^{2}$ in $\left.\sim 72 \mathrm{~h}\right)$, the medium was removed and cells were accurately washed with phosphate-buffered saline, followed by incubation for the desired time (12-144 h) in the appropriate medium. Three dishes were set up for each experimental point. When cells were grown in the absence of glutamine, non-essential amino acids were not added. At each time point, for each dish, the culture medium was collected and the cells were trypsinized and resuspended with the same culture medium, to recover all the cells of the culture. An aliquot of the cell suspension was counted using Trypan blue staining to determine the total number of viable and dead cells. Experiments were repeated at least three times. Cell samples were then used for protein analysis.
The MTS assay was performed using the CellTiter $96^{\circledR}$ AQueous One Solution Cell Proliferation Assay (Promega Corporation, Madison, WI, USA) according to the manufacturer's instructions. Briefly, 7,500 cells/well were seeded in a 96 multi-well dish in complete medium, and after $72 \mathrm{~h}$ the medium was changed and cells were incubated with the appropriate medium (triplicates were set up for each experimental point). To test viability, the CellTiter solution was added to glucose-starved cells $45 \mathrm{~h}$ after starvation, and to glucose- and glutamine-starved cells $13 \mathrm{~h}$ after starvation. In both cases, the solution was added in parallel to cells fed with complete medium. Cell samples were then incubated for a further $3 \mathrm{~h}$ at $37^{\circ} \mathrm{C}$ before measuring absorbance at $492 \mathrm{~nm}$ with the microplate reader (EZ Read 400; Biochrom Ltd., Cambridge, UK). Absorbance values were considered as a measure of viable cells in each sample.

The numbers of viable and dead cells in starved samples and after recovery in complete medium were compared by ANOVA performed using R version 3.5.2 (https://www.r-project.org/).

Olaparib treatment. To inhibit PARP-1 activity, cells were seeded as described above, and incubated for $24 \mathrm{~h}$ in complete DMEM or in glutamine-free medium, with or without addition of 10 or $20 \mu \mathrm{M}$ olaparib (Selleck Chemicals, Houston, TX, USA). At the end of the incubation, cells were collected, counted and analyzed for PARP-1 expression and protein poly(ADP-ribo)sylation (PARylation).

Immunofluorescence. To detect S-phase cells, $5 \times 10^{4}$ cells/well were seeded on coverslips in 12-well plates (Corning Inc., Corning, NY, USA) in complete medium. After $72 \mathrm{~h}$, the medium was replaced with either complete medium or medium without glutamine, and the cells were incubated for 24,48 or $72 \mathrm{~h}$. During the last $30 \mathrm{~min}$ of incubation, $10 \mathrm{mM}$ bromodeoxyuridine (BrdU) was added to each dish. The cells were then fixed and permeabilized on ice with methanol for $4 \mathrm{~min}$, treated with $2 \mathrm{~N} \mathrm{HCl}$ for $20 \mathrm{~min}$ and then incubated with $0.1 \mathrm{M}$ sodium tetraborate $(\mathrm{pH} \mathrm{8.5)}$ ) for $5 \mathrm{~min}$. Following incubation with the primary antibody against BrdU (cat. no. 347580; Becton, Dickinson and Company, Franklin Lakes, NJ, USA) diluted at 1:250, the cells were incubated with a TRITC-labeled secondary antibody (cat. no. 115-025-146; Jackson ImmunoResearch Laboratories, Inc., West Grove, PA, USA) diluted at 1:100. The slides were examined with the Olympus microscope IX71 (Olympus Corp., Tokyo, Japan).

Western blotting. Whole-cell lysates for western blot analysis were prepared using RIPA lysis buffer [1\% Nonidet P40, $50 \mathrm{mM}$ Tris- $\mathrm{HCl} \mathrm{pH} 8.0,150 \mathrm{mM} \mathrm{NaCl}, 0.1 \%$ SDS, $0.1 \%$ DOC, $1 \mathrm{X}$ protease inhibitor cocktail (Thermo Fisher Scientific, Inc., Waltham, MA, USA)], and 1X phosphatase inhibitor cocktail (Roche Diagnostics, Basel, Switzerland)]. The following antibodies were used: anti-PARP-1 (cat. no. ab191217; Abcam, Cambridge, UK) diluted at 1:1,000; anti-poly(ADP-ribose) chains (cat. no. sc-56198; Santa Cruz Biotechnology Inc., Dallas, TX, USA) diluted at 1:1,000; anti-H2AX (cat. no. ab11175; Abcam) diluted at 1:5,000; anti- $\gamma-\mathrm{H} 2 \mathrm{AX}$ (cat. no. JBW301; EMD Millipore, Billerica, MA, USA) diluted at 1:5,000; anti-caspase 9 (cat. no. ALX-210-816; Enzo Life Sciences Inc.) diluted at 1:500, it recognizes the 
caspase 9 cleaved active form of $37 \mathrm{kDa}$; anti-caspase 3 (cat. no. ALX-210-807; Enzo Life Sciences Inc.) diluted at 1:500, it recognizes the caspase 3 cleaved active form of 19 kDa; anti-LC3 (cat. no. 2775; Cell Signaling Technology) diluted at 1:1,000; anti-p62 (cat. no. BML-PW9860; Enzo Life Sciences) diluted at 1:1,000; anti-beclin (cat. no. 3738; Cell Signaling Technology, Inc.) diluted at 1:1,000; anti- $\gamma$-tubulin (cat. no. T6557; Sigma-Aldrich; Merck KGaA, Darmstadt, Germany), diluted at 1:10,000. All primary antibodies were probed by a secondary horseradish peroxidase-conjugated antibody (anti-mouse, cat. no. 115-035-146; anti-rabbit, cat. no. 111-035-144; Jackson ImmunoResearch Laboratories, Inc.). Chemiluminescent assay was used for detection (Clarity $^{\mathrm{TM}}$; Bio-Rad Laboratories, Inc., Hercules, CA, USA). To determined H2AX phosphorylation levels, the intensity of the band in each sample was quantified using the Quantity One 4.6.6 software (Bio-Rad Laboratories Inc.), corrected for the intensity of the corresponding band obtained with the anti-H2AX antibody and then normalized relatively to the appropriate control sample.

\section{Results}

Cellular response to glutamine and/or glucose deprivation. The results obtained from growing cen3tel cells in the absence of glutamine and/or glucose for different time intervals (12-144 h) are presented in Fig. 1A-C. Between 12 and $20 \mathrm{~h}$ of culture, cells deprived of glutamine or glucose showed reduced growth compared with the control cells. Cells starved for both glucose and glutamine had become detached from the bottom of the dish at $16 \mathrm{~h}$ after deprivation, floating as large sheets in the medium (Fig. 1D). However, despite all the cells had lost adhesion to the bottom of the dish, only $36 \%$ of them were positive to Trypan blue, suggesting that cell death followed cell detachment; in fact, after an additional $4 \mathrm{~h}$ of deprivation, the percentage of dead cells increased to $60 \%$ (Fig. 1A). To confirm the presence of viable cells among the detached cells, the MTS assay was performed in cells starved for glucose for $16 \mathrm{~h}$, demonstrating the actual reduction of MTS to formazan in these samples (data not shown).

These results indicate that short term glutamine or glucose starvation reduces cell growth, while the absence of both nutrients leads to cell detachment from the dish and, subsequently, to cell death.

Upon prolonging cell culture up to $144 \mathrm{~h}$ (Fig. 1B and C), we observed that cells propagated in complete medium grew actively for up to $48 \mathrm{~h}$ of incubation, when reached a very high density $\left(\sim 8 \times 10^{5}\right.$ cells $\left./ \mathrm{cm}^{2}\right)$, then remained stable in number and started to die after $96 \mathrm{~h}$, likely because of medium exhaustion due to their high number. The percentage of dead cells markedly increased at subsequent time points, being $>90 \%$ at $144 \mathrm{~h}$ (Fig. 1C). Of note, at that time point, many cells remained attached to the dish, despite they were mostly positive to Trypan blue staining, whereas detached cells floated as single cells (data not shown).

Cells grown in the absence of glutamine remained viable and stable in number up to $72 \mathrm{~h}$, indicating that glutamine starvation led to an arrest in cell growth. This was confirmed by immunofluorescence on cells incubated with BrdU, which demonstrated that the percentage of cells in the S-phase at $24 \mathrm{~h}$ after starvation was lower compared with that in control cells ( $\sim 14$ vs. $\sim 50 \%$, respectively) and decreased to 1.7 and $<1 \%$ at 48 and $72 \mathrm{~h}$ after starvation, respectively. From $96 \mathrm{~h}$ of incubation in the absence of glutamine, the total number of cells remained almost stable, but the number of dead cells increased to a percentage of $\sim 50 \%$ (Fig. 1C). As observed in control cells, at these time points, the majority of cells remained adherent to the dish despite the high percentage of dead cells.

At $\sim 48 \mathrm{~h}$ after glucose deprivation, all the cells had become detached from the bottom of the dish; however, the majority remained alive, with a percentage of dead cells $\sim 30 \%$ (Fig. 1B). The presence of viable cells among the detached cells was again confirmed using the MTS assay (data not shown). This finding was similar to that observed in glucose- and glutamine-starved cells at shorter times of deprivation. At $24 \mathrm{~h}$ of deprivation, cells starved for both nutrients were completely detached from the dish, as expected, and the percentage of dead cells had increased to $70 \%$ (Fig. 1B).

Based on these results, it may be concluded that glutamine deprivation is associated with a prolonged proliferation arrest in cen3tel cells. Deprivation of both glucose and glutamine rapidly led to cell detachment and cell death; the cen3tel response to glucose starvation was similar to that observed in the absence of both glucose and glutamine, albeit with a slower kinetics.

Starved cell response after recovery in complete medium. Whether the cellular response observed after nutrient deprivation was reversible upon supply of complete medium was next investigated. As shown in Fig. 2A, cells starved for either glutamine or glucose for $24 \mathrm{~h}$ were able to restart proliferating once nutrients were supplied. In both conditions, cells performed $\sim 0.8$ PDs every $24 \mathrm{~h}$ up to $48 \mathrm{~h}$ of recovery, after which time proliferation slowed down ( 0.2 PDs between 48 and $72 \mathrm{~h}$ ), likely because of the high cell density in the dish.

Given that cells starved for glutamine remained viable and stable in number for up to $72 \mathrm{~h}$, we tested whether they were also able to resume growing after a 72 -h starvation period (Fig. 2B). At $24 \mathrm{~h}$ after recovery, there was a small, but not statistically significant, increase in the number of viable cells and a mild but statistically significant decline in the number of dead cells (dead cells in 24-h recovery sample vs. dead cells in 72-h starved samples $\mathrm{P}=0.0305$ ); when starved cells were recovered in complete medium for $72 \mathrm{~h}$, the number of viable cells clearly increased (cells performed $\sim 1.3$ PDs) and the number of dead cells was negligible. This behavior was completely different compared with cells that were continuously starved for glutamine for $144 \mathrm{~h}$, which exhibited a net increase in dead cells, as expected (Fig. 2C, middle column).

Thus, cells grown for $72 \mathrm{~h}$ in the absence of glutamine are able to restart growing when nutrients are replenished, although with slower kinetics compared with cells deprived of glutamine for $24 \mathrm{~h}$.

We also investigated whether cells starved for glucose, which were detached from the bottom of the dish but were mostly negative for Trypan blue staining, were committed to die or were able to restart proliferating when recovered in complete medium (Fig. 2D). Cells starved for glucose for $40 \mathrm{~h}$ 
A

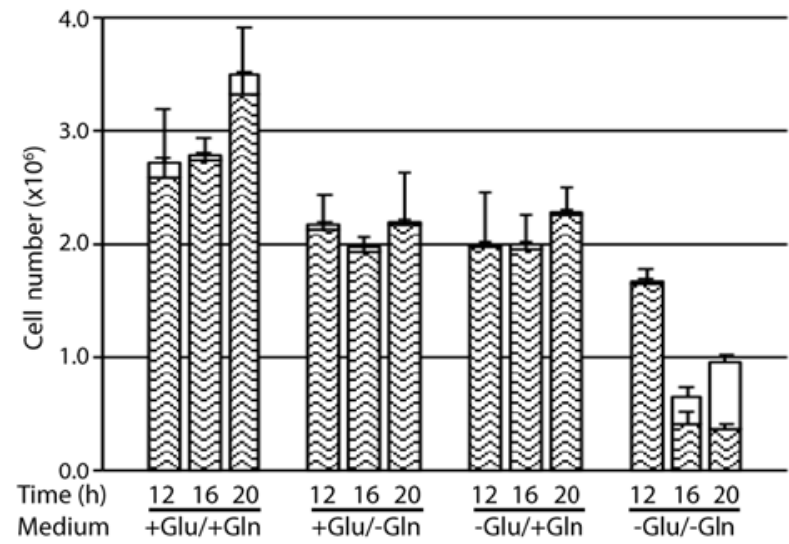

C

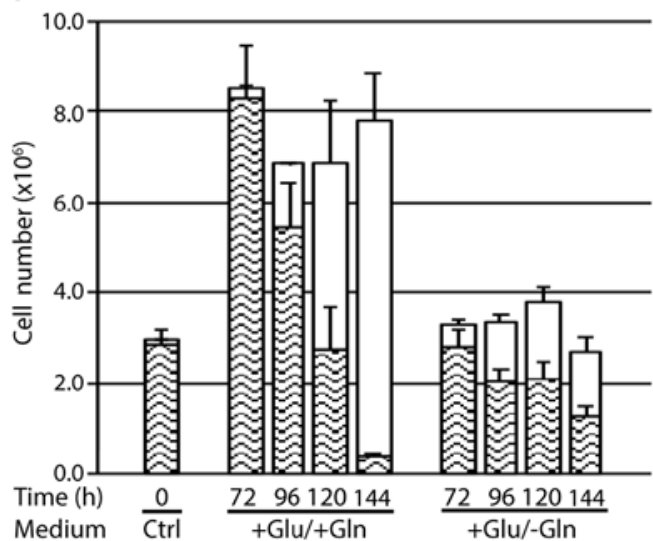

B

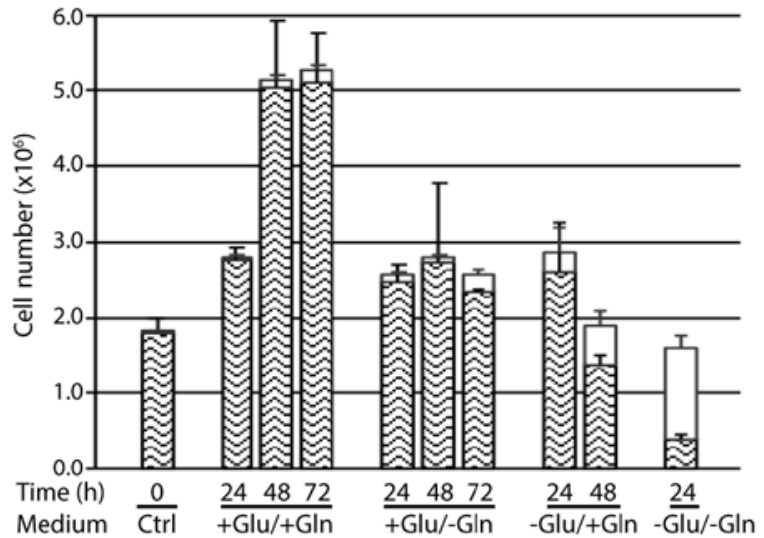

D

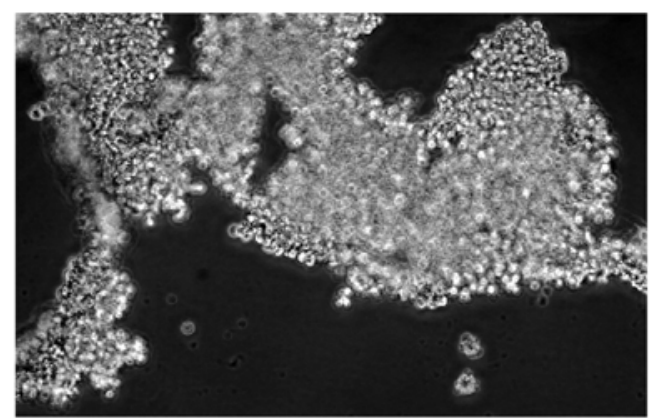

Figure 1. Response of cen3tel cells to glutamine and/or glucose starvation. (A-C) Results obtained in representative experiments are shown. In each plot, the mean number of viable cells (lower part of each column) and dead cells [upper part of each column (white box)] obtained in three replicates is reported for each sample, together with the standard deviations (bars). (D) Cell sheets detached from the bottom of the dish after $16 \mathrm{~h}$ of glutamine and glucose starvation. The image was captured with a $x 4$ objective with the Olympus microscope IX71 equipped with the CoolSnap CCD camera (Teledyne Photometrics, Tucson, AZ, USA), using MetaMorph 7.7.5 software (Molecular Devices, San Jose, CA, USA). Glu, glucose; Gln, glutamine.

were collected by simply washing the dish with the culture medium and, after centrifugation $(400 \times \mathrm{g})$, were replated in complete medium. A 24-h recovery in complete medium had little effect on starved cells; in fact, the number of viable cells was approximately the same as in the starved samples, whereas there was a small, albeit not statistically significant, increase in the number of dead cells. By contrast, at $48 \mathrm{~h}$ after recovery, the number of viable cells increased ( 1.4 PDs), and increased even further after $120 \mathrm{~h}$ of recovery ( 2.7 PDs). This suggests that the population of cells detaching from the dish upon glucose deprivation includes cells which can proliferate when the appropriate culture conditions are re-established. However, at each recovery time point, the proportion of dead cells was high ( $40 \%$ at $72 \mathrm{~h}$ of recovery vs. $\sim 5 \%$ in glutamine-starved cells recovering in complete medium; Fig. 2A and B), suggesting that cells starved for glucose are prone to cell death.

Similar results and conclusions were reached testing the recovery capacity of cells after deprivation of both glucose and glutamine. At $17 \mathrm{~h}$ after starvation, the detached cells were collected and reseeded in complete medium (Fig. 2E), and were found to be able to restart proliferating ( $\sim 1.4$ PDs in $72 \mathrm{~h}$ ), but were also found to be prone to cell death (the percentage of dead cells was $\sim 40 \%$ after $72 \mathrm{~h}$ of recovery).
Analysis of autophagic and apoptotic markers in glutamine and/or glucose-deprived cells. To investigate whether autophagy was activated in starved cells, the expression of the autophagic markers LC3-II and p62 was analyzed (21). In control cells, autophagy activation was observed at 120 and $144 \mathrm{~h}$ of incubation, with a relative increase in LC3-II vs. LC3-I and a decrease in p62 levels (Fig. 3, lanes 14 and 17), likely due to medium exhaustion. In cells grown in the absence of glutamine and in the presence of glucose, there were no indications of autophagy; in fact, the levels of p62 remained constant, and there was no increase in LC3-II levels relative to LC3-I at any given time point (Fig. 3, lanes 2, 6, 9, 12, 15 and 18). In the cell samples grown in the absence of glucose but in the presence of glutamine, an increase in the ratio between LC3-II and LC3-I was observed, starting from $48 \mathrm{~h}$ of starvation (Fig. 3, lanes 7, 10, 13, 16 and 19); at the latest stages of deprivation (120 and $144 \mathrm{~h}$ ) the LC3-I band was barely detectable (Fig. 3, lanes 16 and 19). A clear decrease in $p 62$ levels was observed $72 \mathrm{~h}$ after starvation and at the subsequent time points, indicating the occurrence of autophagy (Fig. 3, lanes 10, 13, 16 and 19). In glucose-starved cells, the expression of the autophagic protein beclin-1 was not altered up to $48 \mathrm{~h}$ after starvation (Fig. 3, lanes 3 and 7) and was found to be decreased at later time points (Fig. 3, lanes 10 and 13), suggesting that a beclin-independent 
A

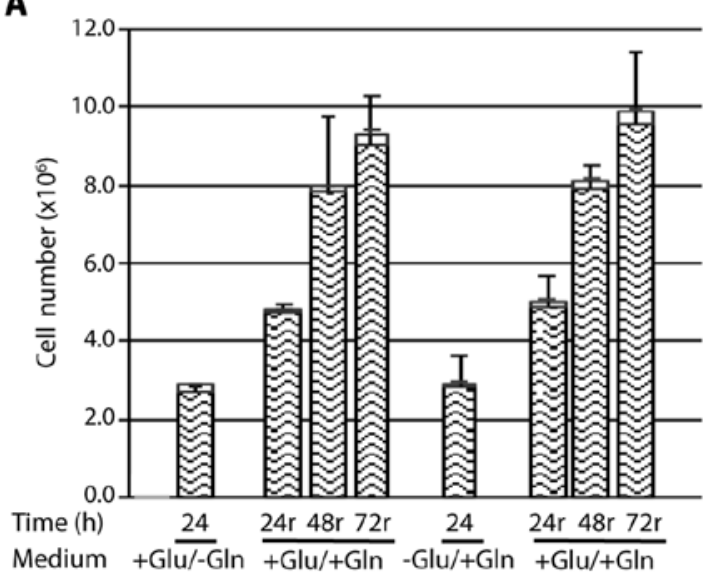

B

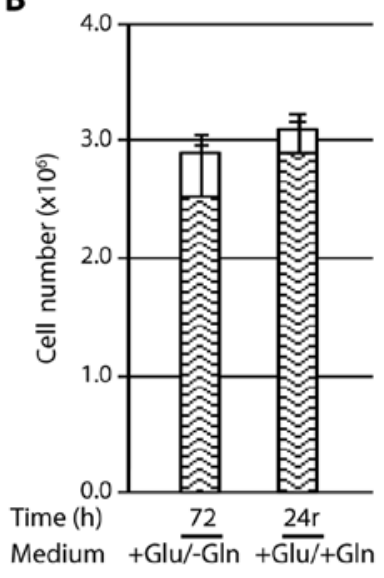

C

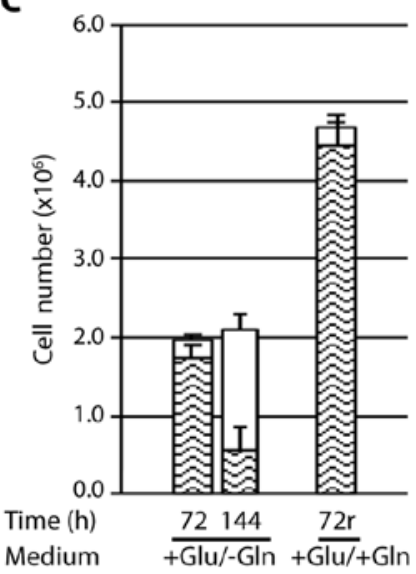

D

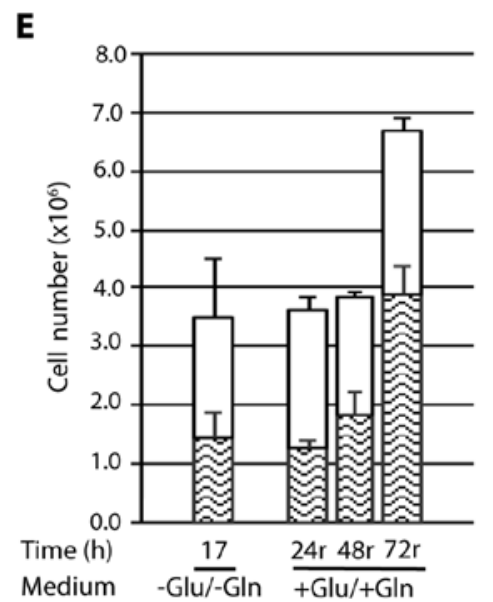

Figure 2. Recovery of starved cen3tel cells in complete medium. In each plot and for each sample, the mean number of viable cells (lower part of each column) and dead cells [upper part of each column (white box)] is reported, together with the standard deviations (bars). In each experiment, the number of cells was counted at the end of the starvation period and after different times of recovery in complete medium (indicated by the number of hours followed by ' $\mathrm{r}$ '). (A) Recovery of cent 3 tel cells starved for glutamine or glucose for $24 \mathrm{~h}$ and then plated in complete medium for 24,48 or $72 \mathrm{~h}$. (B and C) Recovery of cent 3 tel cells starved for glutamine for $72 \mathrm{~h}$ and then plated in complete medium for (B) $24 \mathrm{~h}$ or (C) $72 \mathrm{~h}$. (C) The response to glutamine starvation for $144 \mathrm{~h}$ is also shown (time 144). (D) Recovery of cent3tel cells starved for glucose for $40 \mathrm{~h}$ and then plated in complete medium for 24,48 or $120 \mathrm{~h}$. (E) Recovery of cent 3 tel cells starved for both glucose and glutamine for $17 \mathrm{~h}$ and then plated in complete medium for 24,48 or $72 \mathrm{~h}$. Glu, glucose; Gln, glutamine.

autophagic mechanism (22) was activated in these cells. As was mentioned above, at $48 \mathrm{~h}$ after glucose deprivation the cells were completely detached from the bottom of the dish. However, in cells deprived of both glutamine and glucose for 24 or 48 h, a decrease in p62 levels was observed (Fig. 3, lanes 4, 20 and 21), but it was not paralleled by an increase in the levels of LC3-II (Fig. 3, lanes 4, 20 and 21), suggesting that autophagy was not activated in these cells.

Apoptotic markers, such as the proteolytic fragments of caspase 3, caspase 9 and PARP-1 (23) were then analyzed in starved cells. Cleaved caspase 3 , caspase 9 or the proteolytic band of PARP-1 were not found in any of the cell samples (Figs. 4 and 5), indicating that massive apoptosis does not occur in cen3tel cells starved for glutamine and/or glucose.

However, in several samples a series of bands with a molecular weight higher than PARP-1 were detected by the anti-PARP-1 antibody, suggesting that PARP-1 was modified in those samples. Modified PARP-1 was first observed in cells starved for glutamine. In fact, in the samples grown without glutamine, a smeared PARP-1 signal was already visible at $12 \mathrm{~h}$ after deprivation (Fig. 5A, lane 2). Upon increasing glutamine starvation times, the PARP-1 canonical band became almost undetectable, suggesting that the vast majority of PARP-1 was modified (Fig. 5A, lanes 6, 10, 14, 18 and 21). In the samples deprived of glucose, the smeared signal above the PARP-1 band was barely detectable at $12 \mathrm{~h}$ after starvation and became progressively more intense at longer deprivation time, but the canonical band was visible up to $72 \mathrm{~h}$ of deprivation (Fig. 5A lanes 3, 7, 11, 15 and 19). In these samples the overall intensity of the PARP-1 signals started decreasing $72 \mathrm{~h}$ after deprivation and was not detectable at $96 \mathrm{~h}$ (Fig. 5A, lane 22). In cells deprived of both glucose and glutamine, additional PARP-1 bands were not detected at any time point (Fig. 5A, lanes 4, 8, 12 and 16). PARP-1 modifications were also gradually detected in control cells starting from $16 \mathrm{~h}$ and a subsequent time points (Fig. 5A lanes 5, 9, 13, 17 and 20). In control samples, canonical PARP-1 exhibited a lower degree of modification compared with the corresponding glutamineor glucose-starved specimens.

PARP-1 modification was paralleled by the activation of protein PARylation as shown in western blotting with an antibody against PAR chains (Fig. 5A). Again, protein PARylation occurred at higher levels in glutamine-starved cells (Fig. 5A, lanes 2, 6, 10, 14, 18 and 21) and became 

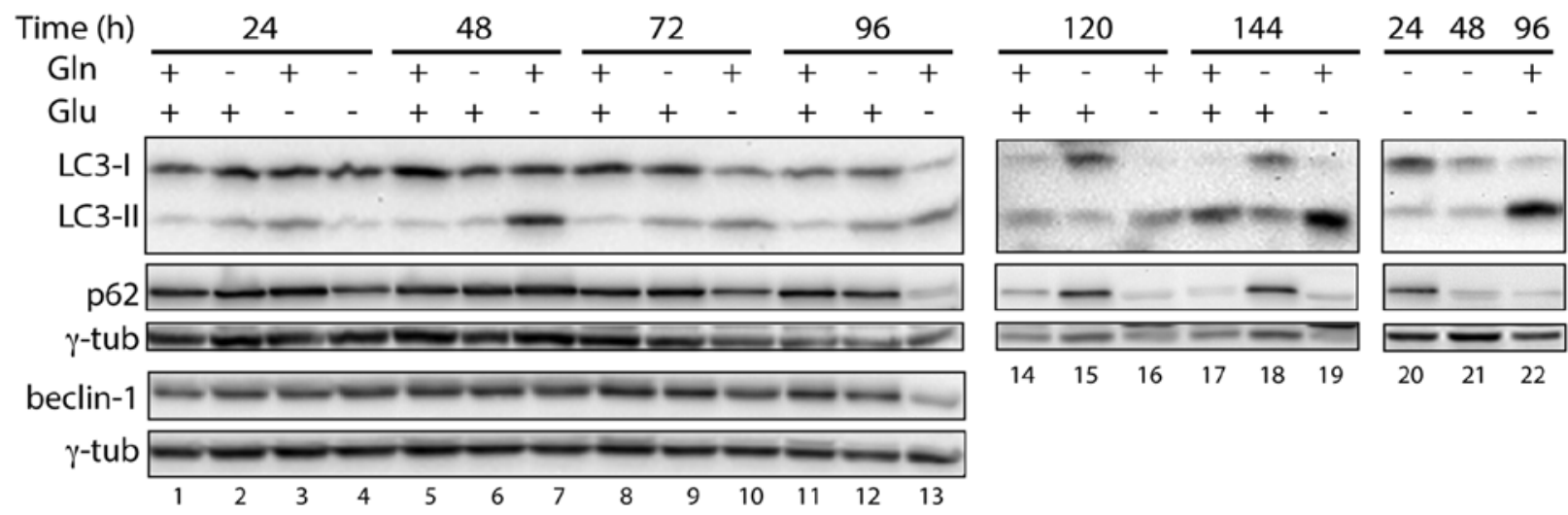

Figure 3. Western blot analysis of the autophagic markers LC3, p62 and beclin-1 in glucose- and/or glutamine-starved cells. The time of incubation of the cells in the different culture media and the media used are indicated above the lanes. The three panels represent results obtained in three independent western blot analyses. Right panel, cells starved for glucose for $96 \mathrm{~h}$ were used for comparison. $\gamma$-tubulin was used as loading control. Glu, glucose; Gln, glutamine.
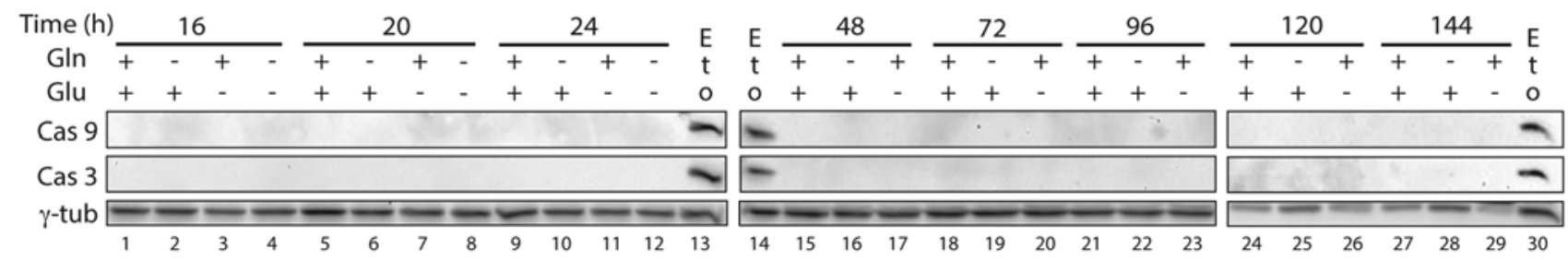

Figure 4. Western blot analysis of the apoptotic markers caspase 9 (Cas 9) and caspase 3 (Cas 3) cleavage fragments in glucose- and/or glutamine-starved cells The time of incubation of the cells in the different culture media and the media used are indicated above the lanes. The three panels represent results obtained in three independent western blot analyses. $\gamma$-tubulin was used as loading control. HeLa cells treated with etoposide (Eto) were used as positive control for caspase activation (lanes 13, 14 and 30). Glu, glucose; Gln, glutamine.

undetectable in glucose starved cells at late time points (72 and 96 h) (Fig. 5A, lanes 19 and 22). In cells starved for both glucose and glutamine protein PARylation was not observable already at the first time point of analysis (12 $\mathrm{h}$ after starvation, Fig. 5A, lane 4). We thus analyzed this protein modification in cells starved for shorter time periods (3, 6 and $9 \mathrm{~h}$ ), finding that glucose and glutamine starvation led to protein PARylation. Under these drastic growth conditions, protein PARylation peaked at $3 \mathrm{~h}$ after starvation, when it was higher than in the other starved samples (Fig. 5B, lane 4 vs. lanes 2 and 3), and then rapidly decreased, becoming lower than in glutamine- or glucose-starved cells at $6 \mathrm{~h}$ after starvation (Fig. 5B, lane 8 vs. lanes 6 and 7) and undetectable at 9 h (Fig. 5B, lane 12). The same trend was observed for PARP-1 modification in cells grown in the absence of both nutrients (Fig. 5B, lanes 4, 8 and 12). Cells grown without glutamine exhibited a clear PARP-1 modification already at 6 and $9 \mathrm{~h}$ after starvation, and to a greater extent compared with the other starved samples (Fig. 5B, lanes 6 and 10).

Given that PARP-1 activation is linked to its auto-PARylation, we tested whether the multiple PARP-1 isoforms detected by western blotting corresponded to PARylated PARP-1 by exposing glutamine-starved cells to the PARP inhibitor olaparib $(24,25)$. As shown in Fig. 5C, olaparib led to the loss of protein PARylation, confirming its activity as a PARP inhibitor (Fig. 5C lanes 5 and 6 vs. lane 4). In glutamine-deprived cells, PARP-1 was highly modified and olaparib treatment led to a concentration-dependent decrease in the smeared signal, with a parallel increase in the intensity of the canonical PARP-1 band (Fig. 5C, lanes 5 and 6 vs. lane 4), confirming the hypothesis that post-translationally modified PARP-1 corresponds to PARylated PARP-1.

Nutrient starvation and DNA DSBs. The results thus far indicated that nutrient starvation induces PARP-1 activation. As PARP-1 is activated by different types of DNA damage, including DNA DSBs, we tested whether cell growth in nutrient-deficient media induced the formation of DSBs. We thus analyzed the DSB marker $\gamma$-H2A histone family member X ( $\gamma$-H2AX) (26) by western blotting in samples starved for different time intervals. Representative western blotting results are shown in Fig. 6A and the results of the quantification of the signal intensities are plotted in Fig. 6B.

Of the cells grown in complete medium, $\gamma$-H2AX levels gradually increased from $48 \mathrm{~h}$ and reached the highest levels at $96 \mathrm{~h}$ ( $\sim \mathrm{x} 3$ compared with control cells; Fig. 6A, lanes 27, 31 and 35 ). In the absence of glutamine, H2AX phosphorylation started to increase $\sim 48 \mathrm{~h}$ after deprivation and dramatically increased at 72 and $96 \mathrm{~h}$ (Fig. 6A, lanes 28, 32 and 36; $\mathrm{x} 5, \mathrm{x} 30.5$ and x62.8, respectively, compared with control cells). In glucose-deprived cells, $\mathrm{H} 2 \mathrm{AX}$ phosphorylation was always lower than in control cells up to $24 \mathrm{~h}$ (Fig. 6A, lanes 3, 6, 9, 16, 20 and 24; between $x 0.2$ and $\times 0.7$, compared with control cells). Between 48 and $96 \mathrm{~h}$ of starvation, phosphorylation levels increased up to approximately x 10 compared to control cells (Fig. 6A, lane 29, 33 and 37). Finally, starvation for glutamine and glucose led to an early decrease in $\gamma$-H2AX 
A
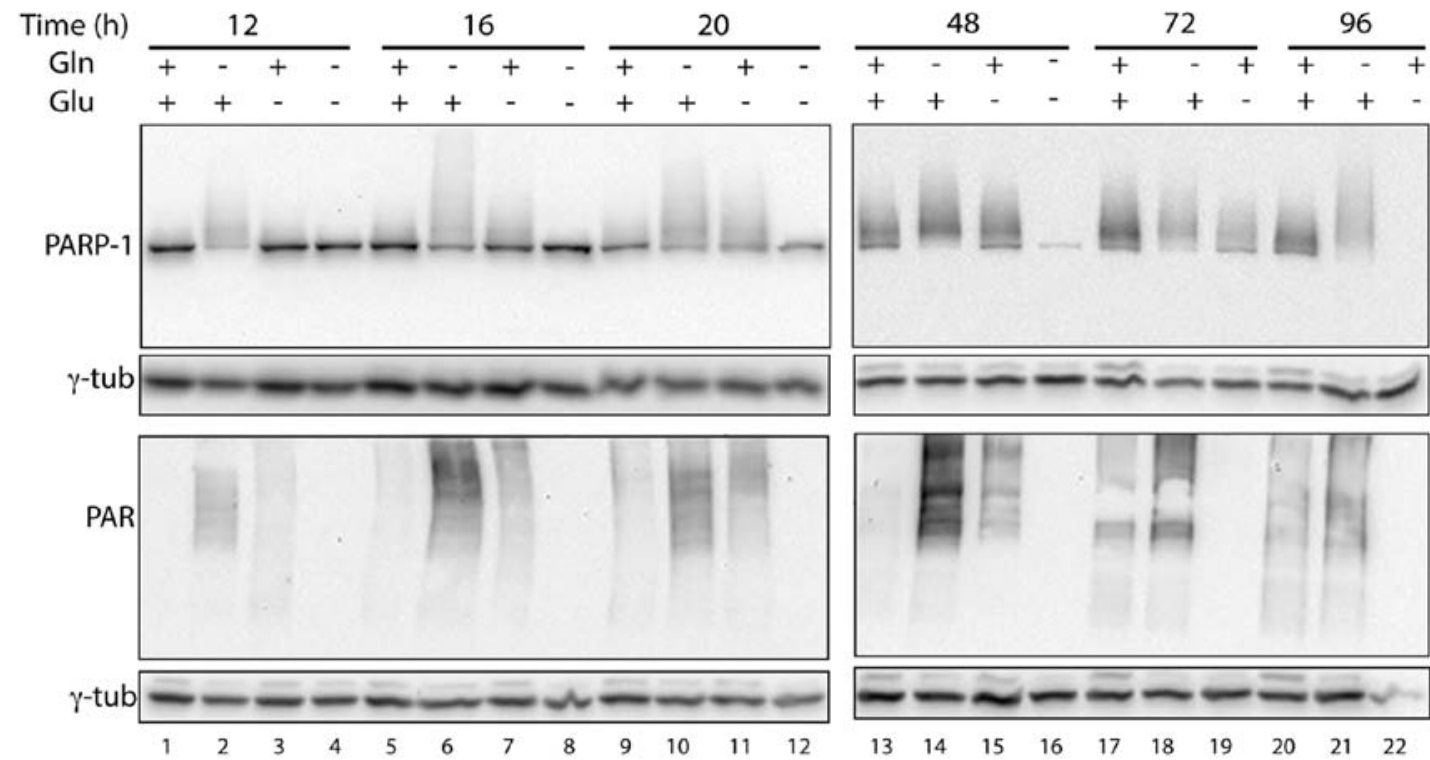

B
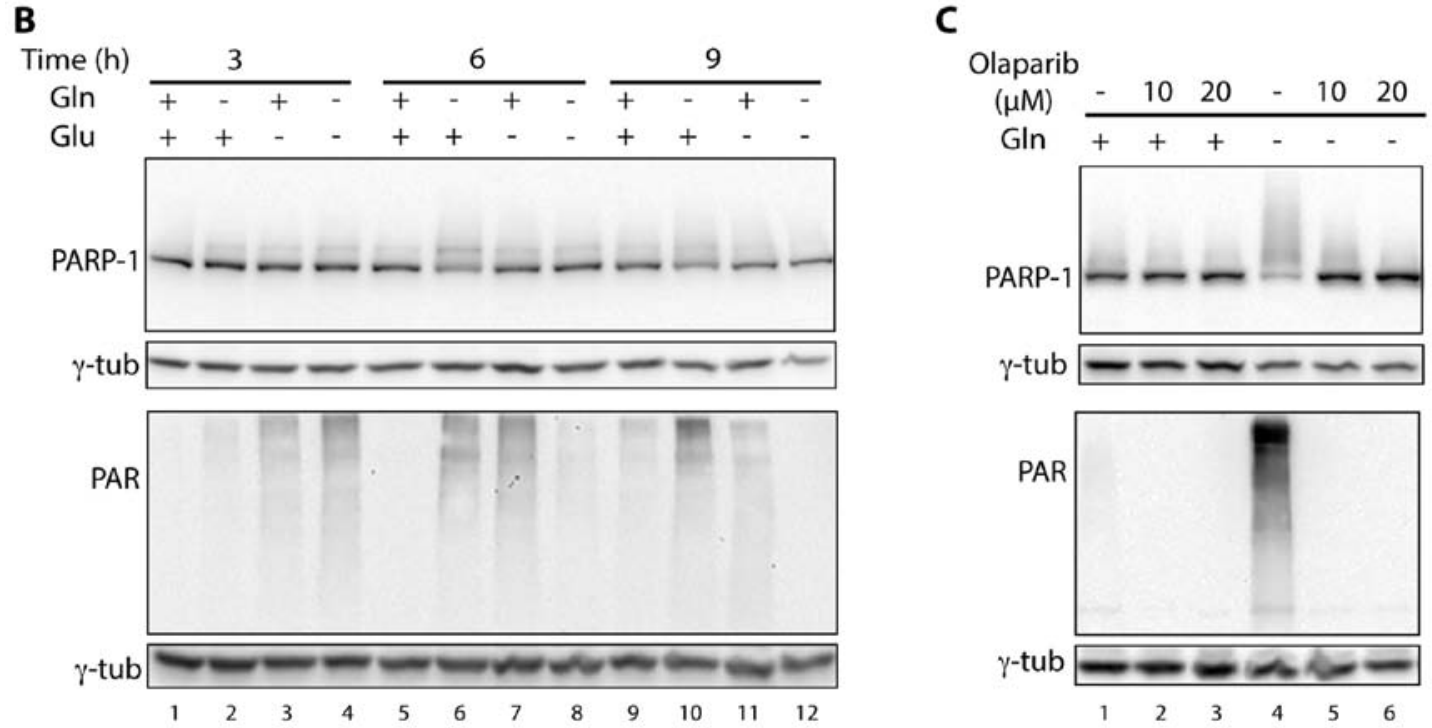

Figure 5. Western blot analysis of PARP-1 expression and PAR levels in (A and B) cen3tel cells starved for glutamine and/or glucose and (C) in glutamine-starved cells exposed to olaparib. The medium composition and the time of incubation are indicated above the lanes. Each panel represents results obtained in three independent western blot analyses. $\gamma$-tubulin was used as loading control. PARP, poly(ADP-ribose) polymerase; Glu, glucose; Gln, glutamine.

levels (3 and $6 \mathrm{~h}$ after starvation, Fig. 6A, lanes 4 and 8; $\mathrm{x} 0.4$ and $\mathrm{x} 0.2$, respectively, compared with control cells) followed by an increase from $16 \mathrm{~h}$ after deprivation (Fig. $6 \mathrm{~A}$, lane 17; x4.1 compared with control cells) and similar levels of phosphorylation at the subsequent time points $(20,24,48$ and $72 \mathrm{~h}$ ) (Fig. 6A, lanes 21, 25, 30 and 34; x2.5, x3.0, x3.2 and $\mathrm{x} 4.2$, respectively, compared with control cells).

Taken together, these results indicate that long-term glutamine deprivation is associated with high levels of $\gamma$-H2AX, while glucose deprivation alone and glucose-glutamine deprivation led to lower levels of $\gamma-\mathrm{H} 2 \mathrm{AX}$ compared with glutamine deficiency. Moreover, this analysis revealed that there was no correlation between PARP-1 activation and $\gamma$-H2AX levels. In fact, PARP-1 activation occurred earlier than $\mathrm{H} 2 \mathrm{AX}$ phosphorylation after nutrient deprivation, indicating that PARP-1 activation was not triggered by DNA DSBs.

\section{Discussion}

Given the importance of glucose and glutamine metabolism for tumor cell survival, nutrient withdrawal may contribute to the suppression of tumor cell proliferation and, eventually, cancer progression. Thus, it is important to explore how different tumor cells respond to nutrient deprivation. In the present study, a well-characterized in vitro cellular system of human transformed fibroblasts was used to compare the cellular and molecular response to deprivation of either glucose or glutamine, or both.

The first effect of starvation for each nutrient was a slowdown in cellular proliferation. This was protracted for $>72 \mathrm{~h}$ after glutamine deprivation, while cells grown in the absence of glucose detached from the bottom of the dish as floating sheets within $48 \mathrm{~h}$ after starvation. Withdrawal of both glucose and glutamine caused cell detachment more quickly compared 
A
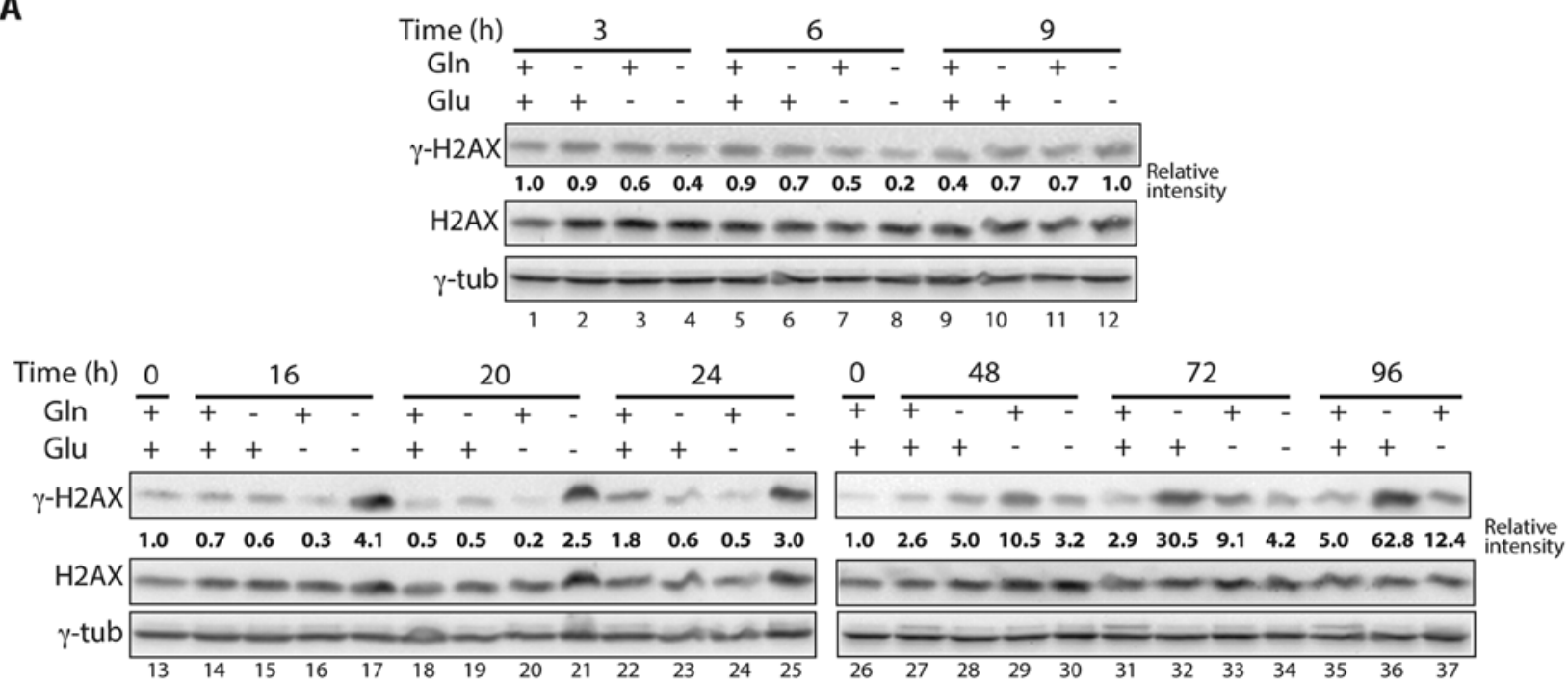

B

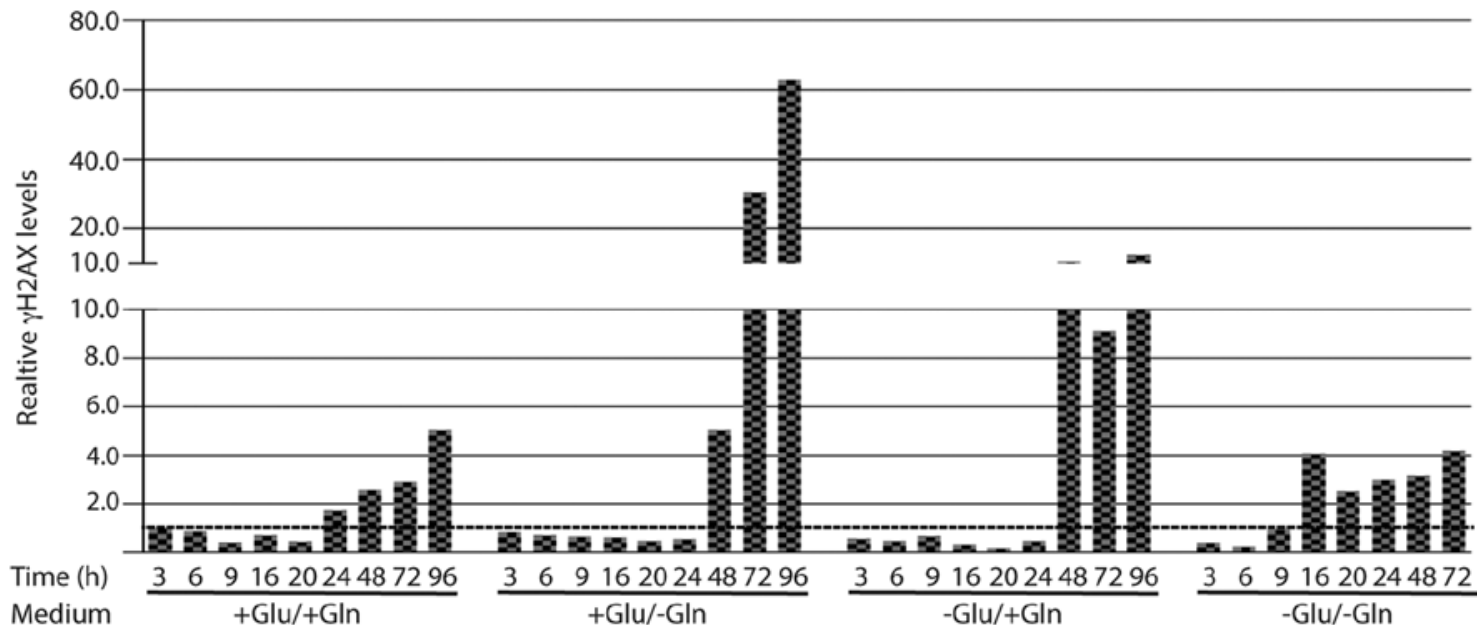

Figure 6. H2AX phosphorylation in cen3tel cells starved for glutamine and/or glucose. (A) Western blot analysis of $\gamma$-H2AX levels in starved cells. The medium composition and the time of incubation are indicated above the lanes. The intensity of each band, corrected for the intensity of the corresponding band obtained with the anti-H2AX antibody, and normalized vs. the appropriate control (the sample at $3 \mathrm{~h}$ for the 3-9-h samples and the samples at t0 in the subsequent blots) is shown below the lanes. $\gamma$-tubulin was used as loading control. (B) Plot summarizing the $\gamma$-H2AX levels relative to control cells in the different samples. The dashed black line is drawn at the value 1. $\gamma$-H2AX, $\gamma$-H2A histone family member X; Glu, glucose; Gln, glutamine.

with glucose starvation alone (within $16 \mathrm{~h}$ ). Under both conditions, a high percentage of floating cells was negative to Trypan blue staining, suggesting that cell detachment occurred prior to cell death. This was confirmed by the fact that some of the detached cells were able to resume proliferation when cultured in complete medium. However, a high percentage of dead cells was also found during the recovery period, possibly because the damage induced by nutrient starvation made cells prone to death. The observation that, in the absence of glucose, cells first detached from the growth substrate and then died, suggests that glucose absence reduces the cells' capacity to adhere to the extracellular matrix and, once in suspension, cells cannot survive due to the metabolic impairment. It is worth mentioning that cen3tel cells are actually able to grow in suspension under non-adherent culture conditions and in the presence of nutrients $(13,27)$. An old study by McGuire (28) reported a similar result, showing that liver cells exposed to glucose metabolism inhibitors lost their adhesion capacity. Under those conditions, the cells remained viable and viability could be rescued by eliminating the inhibitor. The mechanism through which glucose deprivation impairs cell adhesion remains to be fully elucidated. There is evidence that glutamine deprivation can decrease melanoma cell attachment to the extracellular matrix (29); this was not the case in our cellular system. In fact, upon glutamine deprivation only, cells mostly died prior to detaching from the dish or immediately after detachment. However, removal of glucose and glutamine accelerated the detachment process observed in the absence of glucose, suggesting that the metabolic impairment due to glutamine withdrawal acts synergistically with glucose in reducing cell adhesion.

In the literature, the activation of different cell death pathways, mainly apoptosis and autophagy, has been reported in cells under glucose or glutamine deprivation, indicating that different cell types can respond in different ways to nutrient withdrawal, possibly depending on their genetic background (10-12). In glutamine-starved cen3tel cells, no detectable levels of activated caspase 3 and caspase 9 or the 
proteolytic PARP-1 fragment were observed, indicating that apoptosis is not a process massively induced in these cells by glutamine withdrawal. In contrast to what has been reported in the literature (30), glutamine withdrawal did not even activate autophagy, either as a rescue mechanism at the earliest stages of deprivation or as a death pathway at the latest stages. Under our experimental conditions, the main response to glutamine starvation appeared to be growth arrest, followed by caspase-independent cell death only after prolonged starvation (120-144 h). In glucose-starved cells, autophagy activation was detected, starting at $48 \mathrm{~h}$ of deprivation, when all the cells had become detached from the bottom of the dish. Autophagy activation has been described under glucose deprivation conditions and is mainly due to ATP depletion (30). Furthermore, autophagy activation was found in control cells, occurring at the latest time points of analysis (120 and $144 \mathrm{~h}$ ), when the percentage of dead cells was very high (>90\% at $144 \mathrm{~h}$ ); thus, in this context, autophagy acts as a death mechanism and is not activated to prolong cell survival during medium exhaustion. When cells were grown in the absence of both glucose and glutamine, and became extensively detached from the dish, we did not identify markers of either apoptosis or autophagy. Under these conditions, cells may activate a non-canonical mechanism of death, due to the highly impaired metabolism.

PARP-1 analysis in the different cell samples revealed, in addition to the expected PARP-1 band, the presence of a series of bands suggestive of proteins of molecular weight higher than PARP-1 that were able to react with the antibody. It is well known that, in response to several stimuli, including DNA damage, PARP-1 is activated and becomes able to catalyze the PARylation of itself and several other proteins using $\mathrm{NAD}^{+}$as a substrate (31). As much of the PARP-1 activity is self-directed (31), the additional PARP-1 bands detected by western blotting may correspond to PARylated PARP-1. Indeed, we found that PARP-1 modifications disappeared upon treatment of glutamine-starved cells with the PARP-1 inhibitor olaparib, demonstrating that they were due to auto-PARylation. At equal deprivation times, glutamine-starved cells exhibited more prominent PARP-1 auto-modification, as well as higher protein PARylation levels, compared with glucose-starved cells. By contrast, in cells grown in the absence of both glucose and glutamine, PARP-1 and cellular proteins were PARylated only at very early times after starvation ( 3 and $6 \mathrm{~h}$ ). The lack of PARylation at later times may be attributed to the dramatic decrease in the levels of the substrate $\mathrm{NAD}^{+}$, due to a deep impairment in the energy metabolism under highly stressful conditions.

Evidence indicates that nutrient deprivation stimulates the formation of reactivate oxygen species (ROS), which can in turn induce DNA double-strand breaks (DSBs) (32-34). Analyzing DNA DSBs through $\mathrm{H} 2 \mathrm{AX}$ phosphorylation in different cancer cells lines, Tran et al (34) reported high levels of $\gamma-\mathrm{H} 2 \mathrm{AX}$ already $24 \mathrm{~h}$ after glutamine deprivation and prior to cell death. We herein demonstrated that $\mathrm{H} 2 \mathrm{AX}$ phosphorylation is not rapidly induced by glutamine and/or glucose deprivation, but occurs when cellular viability is highly compromised. $\gamma$-H2AX reached its highest levels in glutamine-starved cells compared with cells under different growth conditions. However, it has to be pointed out that $\mathrm{H} 2 \mathrm{AX}$ phosphorylation was detected in glucose- or double-deprived cells after they had already become detached from the bottom of the dish, and it may be less efficient in carrying out this modification after prolonged detachment. In glucose- and double-starved cells, $\gamma$-H2AX levels were actually reduced compared with those in the control cells at the earlier stages of starvation. As hypothesized by Hruda et al (35), this may be due to the high glycolytic and mitochondrial metabolic activities that produce high ROS levels in cells grown in the presence of high glucose concentration.

Under all nutrient deprivation conditions, H2AX phosphorylation was not found to correlate with either protein PARylation or PARP-1 PARylation, PARylation always preceded the increase in $\gamma-\mathrm{H} 2 \mathrm{AX}$ levels. In particular, in cells starved for both glucose and glutamine, protein PARylation was detected only early after starvation, when the $\gamma-\mathrm{H} 2 \mathrm{AX}$ levels were lower compared with those in control cells. Thus, other types of DNA damage rather than DSBs are likely responsible for PARP-1 activation after glucose and/or glutamine starvation.

Recently, Aguilera-Gomez et al (36) reported that protein mono(ADP)-ribosylation in Drosophila cells is crucial for Sec body formation and cell survival. Sec bodies are pro-survival cytoplasmic assemblies produced in Drosophila cells upon amino acid starvation. Despite the counterpart of Sec bodies not being present in mammalian cells, it cannot be excluded, as Aguilera-Gomez et al (36) hypothesized, that mammalian PARPs activated upon glutamine deprivation may act in concert to PARylate different target proteins to promote the formation of assemblies similar to Sec bodies, which may improve cell survival under metabolic stress.

In conclusion, the results of the present study may widen our current knowledge concerning the consequences of glutamine and or glucose starvation in transformed cells. Glucose deprivation was found to be a harsher condition compared with glutamine starvation. In transformed cells, the absence of glucose rapidly induced a decrease in cell attachment to the substrate and autophagic death. By contrast, glutamine starvation first led to an arrest in cell growth, and cells died only after prolonged absence of glutamine, without activating autophagy or massive apoptosis. The absence of glutamine and/or glucose caused extensive PARP-1 PARylation, which was not associated with $\mathrm{H} 2 \mathrm{AX}$ phosphorylation, suggesting that PARylation is not due to DSBs, at least at the early stages after nutrient deprivation. Moreover, the consequences of glucose or glutamine deprivation were shown to be reversible, indicating that starved tumor cells can recover if nutrient availability in the microenvironment is restored.

\section{Acknowledgements}

Not applicable.

\section{Funding}

No funding was received.

\section{Availability of data and materials}

All data generated or analyzed during this study are included in this published article. 


\section{Authors' contributions}

IC, GP and CMa performed the experiments and analyzed the data. IC and CMo conceived the experiments and wrote the manuscript. All authors read and approved the manuscript and agree to be accountable for all aspects of the research in ensuring that the accuracy or integrity of any part of the work are appropriately investigated and resolved.

\section{Ethics approval and consent to participate}

Not applicable.

\section{Patient consent for publication}

Not applicable.

\section{Competing interest}

The authors declare that they have no competing interests.

\section{References}

1. Hanahan D and Weinberg RA: Hallmarks of Cancer: The next generation. Cell 144: 646-674, 2011.

2. Robey RB, Weisz J, Kuemmerle NB, Salzberg AC, Berg A, Brown DG, Kubik L, Palorini R, Al-Mulla F, Al-Temaimi R, et al: Metabolic reprogramming and dysregulated metabolism: Cause, consequence and/or enabler of environmental carcinogenesis? Carcinogenesis 36 (Suppl 1): S203-S231, 2015.

3. Pavlova NN and Thompson CB: The emerging hallmarks of cancer metabolism. Cell Metab 23: 27-47, 2016.

4. Warburg O: On the origin of cancer cells. Science 123: 309-314, 1956.

5. Daye D and Wellen KE: Metabolic reprogramming in cancer: Unraveling the role of glutamine in tumorigenesis. Semin Cell Dev Biol 23: 362-369, 2012.

6. Jones DP and Sies H: The redox code. Antioxid Redox Signal 23: 734-746, 2015.

7. Altman BJ, Stine ZE and Dang CV: From Krebs to clinic: Glutamine metabolism to cancer therapy. Nat Rev Cancer 16: 619-634, 2016.

8. Wolpaw AJ and Dang CV: Exploiting metabolic vulnerabilities of cancer with precision and accuracy. Trends Cell Biol 28: 201-212, 2018.

9. Fuchs BC and Bode BP: Stressing out over survival: Glutamine as an apoptotic modulator. J Surg Res 131: 26-40, 2006.

10. Yuneva M, Zamboni N, Oefner P, Sachidanandam R and Lazebnik Y: Deficiency in glutamine but not glucose induces MYC-dependent apoptosis in human cells. J Cell Biol 178 93-105, 2007.

11. Shanware NP, Bray K, Eng CH, Wang F, Follettie M, Myers J, Fantin VR and Abraham RT: Glutamine deprivation stimulates mTOR-JNK-dependent chemokine secretion. Nat Commun 5: 4900, 2014.

12. Choi SW, Song JK, Yim YS, Yun HG and Chun KH: Glucose deprivation triggers Protein kinase $C$ Dependent $\beta$-catenin proteasomal degradation. J Biol Chem 290: 9863-9873, 2015.

13. Mondello C, Chiesa M, Rebuzzini P, Zongaro S, Verri A, Colombo T, Giulotto E, D'Incalci M, Franceschi C and Nuzzo F: Karyotype instability and anchorage-independent growth in telomerase-immortalized fibroblasts from two centenarian individuals. Biochem Biophys Res Commun 308: 914-921, 2003.

14. Zongaro S, de Stanchina E, Colombo T, D'Incalci M, Giulotto E and Mondello C: Stepwise neoplastic transformation of a telomerase immortalized fibroblast cell line. Cancer Res 65: 11411-11418, 2005.

15. Belgiovine C, Frapolli R, Bonezzi K, Chiodi I, Favero F, Mello-Grand M, Dei Tos AP, Giulotto E, Taraboletti G,D'Incalci M and Mondello C: Reduced expression of the rOCK inhibitor Rnd3 Is associated with increased invasiveness and metastatic potential in mesenchymal tumor cells. PLoS One 5: e14154, 2010.
16. Ostano P, Bione S, Belgiovine C, Chiodi I, Ghimenti C, Scovassi AI, Chiorino G and Mondello C: Cross-analysis of gene and miRNA genome-wide expression profiles in human fibroblasts at different stages of transformation. OMICS 16: 24-36, 2012.

17. Gao P, Tchernyshyov I, Chang TC, Lee YS, Kita K, Ochi T, Zeller KI, De Marzo AM, Van Eyk JE, Mendell JT and Dang CV: c-Myc suppression of miR-23a/b enhances mitochondrial glutaminase expression and glutamine metabolism. Nature 458: 762-765, 2009.

18. Belgiovine C, Chiodi I and Mondello C: Relocalization of cell adhesion molecules during neoplastic transformation of human fibroblasts. Int J Oncol 39: 1199-1204, 2011.

19. Chiodi I, Belgiovine C, Zongaro S, Ricotti R, Horard B, Lossani A, Focher F, Gilson E, Giulotto E and Mondello C: Super-telomeres in transformed human fibroblasts. Biochim Biophys Acta 1833: 1885-1893, 2013.

20. Belgiovine C, Chiesa G, Chiodi I, Frapolli R, Bonezzi K, Taraboletti G, D'Incalci M and Mondello C: Snail levels control the migration mechanism of mesenchymal tumor cells. Oncol Lett 12: 767-771, 2016

21. Mizushima N, Yoshimori $\mathrm{T}$ and Levine B: Methods in mammalian autophagy research. Cell 140: 313-326, 2010.

22. Codogno P, Mehrpour M and Proikas-Cezanne T: Canonical and non-canonical autophagy: Variations on a common theme of self-eating? Nat Rev Mol Cell Biol 13: 7-12, 2011.

23. Ward TH, Cummings J, Dean E, Greystoke A, Hou JM, Backen A, Ranson M and Dive C: Biomarkers of apoptosis. Br J Cancer 99: 841-846, 2008.

24. Bryant HE, Schultz N, Thomas HD, Parker KM, Flower D, Lopez E, Kyle S, Meuth M, Curtin NJ and Helleday T: Specific killing of BRCA2-deficient tumours with inhibitors of poly(ADP-ribose) polymerase. Nature 434: 913-917, 2005.

25. Farmer H, McCabe N, Lord CJ, Tutt AN, Johnson DA, Richardson TB, Santarosa M, Dillon KJ, Hickson I, Knights C, et al: Targeting the DNA repair defect in BRCA mutant cells as a therapeutic strategy. Nature 434: 917-921, 2005.

26. Kuo LJ and Yang LX: Gamma-H2AX-a novel biomarker for DNA double-strand breaks. In Vivo 22: 305-309, 2008.

27. Bono B, Ostano P, Peritore M, Gregnanin I, Belgiovine C, Liguori M, Allavena P, Chiorino G, Chiodi I and Mondello C: Cells with stemness features are generated from in vitro transformed human fibroblasts. Sci Rep 8: 13838, 2018.

28. McGuire EJ: Intercellular adhesive selectivity. II. Properties of embryonic chick liver cell-cell adhesion. J Cell Biol 68: 90-100, 1976.

29. Fu YM,Zhang H, Ding M, Li YQ, Fu X, Yu ZX and Meadows GG: Specific amino acid restriction inhibits attachment and spreading of human melanoma via modulation of the integrin/focal adhesion kinase pathway and actin cytoskeleton remodeling. Clin Exp Metastasis 21: 587-598, 2004.

30. Galluzzi L, Pietrocola F, Levine B and Kroemer G: Metabolic control of autophagy. Cell 159: 1263-1276, 2014.

31. Gupte R, Liu Z and Kraus WL: PARPs and ADP-ribosylation: Recent advances linking molecular functions to biological outcomes. Genes Dev 31: 101-126, 2017.

32. Scherz-Shouval R, Shvets E, Fass E, Shorer H, Gil L and Elazar Z: Reactive oxygen species are essential for autophagy and specifically regulate the activity of Atg4. EMBO J 26: 1749-1760, 2007.

33. Rodríguez-Vargas JM, Ruiz-Magaña MJ, Ruiz-Ruiz C, Majuelos-Melguizo J, Peralta-Leal A, Rodríguez MI, Muñoz-Gámez JA, de Almodóvar MR, Siles E, Rivas AL, et al: ROS-induced DNA damage and PARP-1 are required for optimal induction of starvation-induced autophagy. Cell Res 22: 1181-1198, 2012.

34. Tran TQ, Ishak Gabra MB, Lowman XH, Yang Y, Reid MA, Pan M, O'Connor TR and Kong M: Glutamine deficiency induces DNA alkylation damage and sensitizes cancer cells to alkylating agents through inhibition of ALKBH enzymes. PLoS Biol 15: e2002810, 2017.

35. Hruda J, Sramek V and Leverve X: High glucose increases susceptibility to oxidative-stress-induced apoptosis and DNA damage in K-562 cells. Biomed Pap Med Fac Univ Palacky Olomouc Czech Repub 154: 315-320, 2010.

36. Aguilera-Gomez A, van Oorschot MM, Veenendaal T and Rabouille C: In vivo vizualisation of mono-ADP-ribosylation by dPARP16 upon amino-acid starvation. Elife 5: e21475, 2016. 